\title{
The role of item fixation in haptic search
}

\author{
Myrthe A. Plaisier* Irene A. Kuling Wouter M. Bergmann Tiest $\quad$ Astrid M.L. Kappers
}

Helmholtz Institute, Utrecht University

The Netherlands

\begin{abstract}
Enclosing objects in the hand is a common and efficient way of haptic exploration. Recently, the importance of grasping for more realistic haptic perception of virtual objects has been recognised in haptic interface design. While several studies on haptic perception have addressed haptic exploration of a single object, perception of several objects grasped together in the hand has received almost no attention yet. In this study we focus on the importance of freedom to manipulate the objects in the hand for three-dimensional shape perception. Furthermore, we investigate differences in detection speed for different positions in the grasping hand. Subjects were asked to search for a cube among spheres or for a sphere among cubes. Response times were measured for different locations of target shape in the hand. Also, the way in which the items were fixed was varied from allowing small displacements and rotation of the shapes to rigidly fixed. There were only differences in search times between the different positions in the hand, when the centre item was difficult to access because of the surrounding items. Finally, we show that search was faster when the items were rigidly fixed than when displacement and rotation was possible. This shows that more exploratory freedom does not necessarily make search for a three-dimensional shape faster.
\end{abstract}

\section{INTRODUCTION}

It has been shown that typical exploratory procedures can be identified for haptically extracting certain types of information. Examples are lateral motion for perceiving roughness, lifting the objects for assessing weight and enclosure for global shape perception [6]. Furthermore, it has been shown that constraining the exploratory movements can impair haptic object recognition [9]. In haptic display design, the importance of haptic exploration using the whole hand and enclosing virtual objects for a more realistic perception of three-dimensional objects has been recognised. Devices like the CyberGrasp enable force-feedback for grasping virtual objects [1]. Also, there are displays being developed that give the sensation that an object is held between two hands by applying pressure and shear forces to the palm [10]. While the importance of haptic perception of objects through grasping objects in the hand has been made clear, relatively few studies have addressed perception of objects enclosed in the hand.

In daily life it is a common situation to have multiple objects grasped together in the hand. We can extract information about the shapes, sizes and materials of the objects in our hand by grasping them and making finger and hand movements. The object positions in the hand can be freely rearranged and the shapes can be rotated over an arbitrary axis. The question arises how important it is that the objects' positions and orientations in the hand can be freely manipulated for extracting information about the objects.

It has also been shown that there is a typical two-stage procedure for exploring an object [7]. First, there is a grasp and lift stage,

*e-mail: M.A.Plaisier@uu.nl which is followed by more detailed exploration like contour following. A similar procedure could be identified for haptic exploration of several three-dimensional shapes grasped together in the hand. After grasping the shapes in the hand, they can be explored more extensively by making hand and finger movements or they can even be explored subsequently if necessary [14]. When exploring several objects simultaneously, exploratory freedom can be constrained by fixing the objects' relative positions and orientations in space. In that case the objects cannot be rearranged in the hand and item manipulation is constrained. This constraint also means that if you are trying to find a particular object among other objects in your hand, this so-called 'target item' may be easier to locate when it makes contact with certain parts of the hand than other parts. There are several factors that might cause differences in detection speed. Firstly, it may depend on the number of nerve endings in the skin of the different parts of the hand. Estimations of the number of nerve endings for different areas of the hand showed that density is relatively high for the fingers and substantially smaller for the palm of the hand [4]. It has also been shown that spatial resolution reported is higher for the fingers than for the palm of the hand [3]. Secondly, when grasping rigid objects, detection speed may also depend on the forces that can be exerted on the objects grasped at different locations in the hand. Finally, response times may also depend on the ability to focus attention to different parts of the hand.

Search tasks have often been used in vision to investigate saliency of certain object features. Typically, the task is to respond as fast as possible whether a certain target item is present among distractor items. Usually, the number of items is varied and response times are measured as a function of the number of items. The slope of this function is then a measure of the efficiency with which the search was performed. This paradigm has been successfully expanded to the haptic domain. In several studies a haptic search task has been used to investigate saliency of several object properties. There are different ways in which the items can be presented. They can be pressed onto the separate fingers $[5,8]$ or they can be presented in a two-dimensional plane $[11,12]$. Recently, we have added a new type of haptic search task in which threedimensional shapes are grasped together in the hand [14]. In this case the shapes were suspended from flexible wires which allowed subjects to freely rotate and translate the shapes. Note that this also allowed the subjects to manipulate the item positions with respect to each other.

This last study showed that search for a target shape with edges among distractor shapes without edges or vice versa is highly efficient under relatively unconstrained item manipulation conditions. While that study aimed at investigating saliency of threedimensional shape features, the present study aims at providing more insight into the influence of constraining translational and rotational freedom of the shapes in the hand on search times. By fixating the items, the subjects' freedom of moving the items in the hand is constrained, while exploratory movements are left unconstrained. This is a new way of constraining exploratory freedom. Constraining exploratory freedom by constraining exploratory movements, has been previously shown to impair haptic information extraction [9]. To investigate whether constraining exploratory freedom by fixating the items leads to an increase in search times, the way in 
which the items were fixed in space was varied. A consequence is that the relative positions of the items cannot be manipulated like in the previous study. For instance, if the target item is initially in the centre it cannot be moved out from among the other items. In order to see how this affects target detection, we investigated differences in search times for different locations of the target shape in the hand. Note that we did not intend to measure sensitivity of different part of the skin on the hand. Rather we investigated processing of information across different positions in the grasping hand. Therefore, we let subjects perform a search task by actively grasping the stimuli and we let exploratory movements largely unconstrained.

In the present study again a search task where the items consisted of three-dimensional shapes was used, but in this case response times were not measured as a function of the number of items. Rather, the number of items was kept constant, while the target position was varied. Response times were thus measured as a function of target position. Furthermore, there were three different ways in which the items could be fixed. One way of fixation allowed for small displacements and rotation of the items. In the two other fixation methods the items were rigidly fixed to a certain position and orientation with all items in the same plane (bottoms levelled) or with the centre item lowered. In this last condition the global shape of the plane in which the items are fixed is curved and corresponds roughly with the shape of the grasping hand, which might reduce response times. The task was either to search for a cube among spheres or for a sphere among cubes. These shapes were chosen because it has been shown that in this case the target can usually be detected through only grasping the items together in the hand without more detailed exploration being necessary [14].

\section{Method}

\subsection{Participants}

Eight paid undergraduate students (mean age $20 \pm 2$ years) participated in the experiment. Four of them were female. Seven subjects were right-handed and one was left-handed according to Coren's test [2]. None of the subjects reported any known hand deficits. Participants were treated in accordance with the local guidelines and gave their informed consent.

\subsection{Stimuli and Apparatus}

The stimuli consisted of brass cubes (edge length $1.5 \mathrm{~cm}$ ) and spheres (radius $0.93 \mathrm{~cm}$ ). These sizes were chosen such that the volume of both shapes was constant to eliminate possible volume and weight cues. The number of items presented was always five and the shapes were spatially located at the positions indicated in Figure 1a. In this figure it can be seen that each position corresponded with a certain region of the hand. Roughly these were: near the thumb (position 1), near the wrist (position 2), near the little finger (position 3), near the middle finger (position 4) and in the centre (position 5). These positions were only roughly defined. Since subjects actively grasped the items, a more precise definition of the item positions is not possible and for the purpose of the experiment this was sufficient.

In some conditions the items were fixed to a metal tube through a rigid connection (Figure 1b). This method of fixation did not allow for movement of the items with respect to each other and did not allow for rotation of the shapes. All items were either fixed in the same plane with the bottoms of the shapes levelled (fixed), or the centre item was lowered $0.5 \mathrm{~cm}$ with respect to the other items (fixed with centre item lowered). The items could also be fixed in a way that did allow for the items to be displaced slightly and to be rotated. This was accomplished by suspending the shape from wires. The wires were pulled through the tubes leaving $0.5 \mathrm{~cm}$ of wire from the bottom of the tube (partially fixed). For each of the three methods of fixation two search tasks were performed. The task was either to find a cube among spheres, or the target could be a sphere among cubes as distractor items. This added up to a total of six conditions.

Response times were recorded using a custom-built response time measuring device. Time measurement was started when the subject touched the stimulus activating the touch sensitive contact of the device. The measurement was terminated with a vocal response registered with a headset microphone. The resulting response time was then recorded with an accuracy of $10 \mathrm{~ms}$ (for technical details about this set-up see [13]).

\subsection{Procedure}

The subjects were blindfolded and they placed their dominant hand with the palm facing upward in a holder prior to the beginning of a trial (Figure 1c). There were also holders for the elbow and for the wrist to keep the starting position of the hand and arm the same prior to each trial. They were instructed to reach upward and grab all items simultaneously. It was also emphasised that they should try to grasp the items in the same way each trial. Other than this there were no restrictions on exploratory hand and finger movements. Each of the subjects could place the fingers in between the tubes to completely enclose all of the shapes simultaneously with the hand. Subjects were instructed to respond whether a target item was present as fast as possible by calling out the Dutch equivalents of 'yes' and 'no'. It was also emphasised that it was important that the answer was correct. The subjects received feedback on whether the answer was correct from the experimenter. Error trials were repeated at the end of the block and only correct responses were included in the analysis.

All conditions were performed in separate blocks of trials and each block of trials was preceded by a training session. To allow subjects to optimise their performance, they were informed prior to the experiment of which shape the target item and which shape the distractor items in that particular block of trials would have. Subjects performed at least 20 training trials and trials were continued until 10 subsequent trials were correct. It was never necessary to exceed 20 training trials. Each condition was performed in a separate block of trials. The target could be located at any of the five item positions. For each target position 10 trials were performed totalling 50 trials. There were also 50 trials in which the target item was absent such that in half of the trials there was a target item present. All of the subjects performed each of the six experimental conditions. Care was taken that the order in which the conditions were performed was as close to counterbalanced over subjects as possible.

\section{REsULtS}

First the target present and target absent trials were analysed regardless of target position. Figure $2 \mathrm{a}$ shows the response times averaged over subjects for target present and target absent trials for each of the conditions. A $3 \times 2 \times 2$ (fixation method $\times$ target shape $\times$ target presence) repeated measures ANOVA (Analysis Of Variance) was performed on the response times from each of the conditions. Sphericity was assumed according to Mauchly's test for all factors unless indicated otherwise. The ANOVA showed main effects for fixation method $\left(F_{2,14}=7.0, p<0.01\right)$, target shape $\left(F_{1,7}=46.4, p<0.001\right)$ and target presence $\left(F_{1,7}=23.0, p<0.01\right)$. Furthermore, the interaction between target shape and target presence was significant $\left(F_{1,7}=13.3, p<0.01\right)$. These results mean that response times were significantly longer for target absent trials than for target present trials. This is commonly found in search tasks. The interaction shows that the difference in response time between target absent and target present trials was larger when the target was a sphere than when it was a cube. Pair-wise comparisons (post-hoc $t$-tests) with Bonferroni correction between the three ways of item fixation showed that the response times were significantly faster when the items were fixed $(p<0.05)$ and fixed 

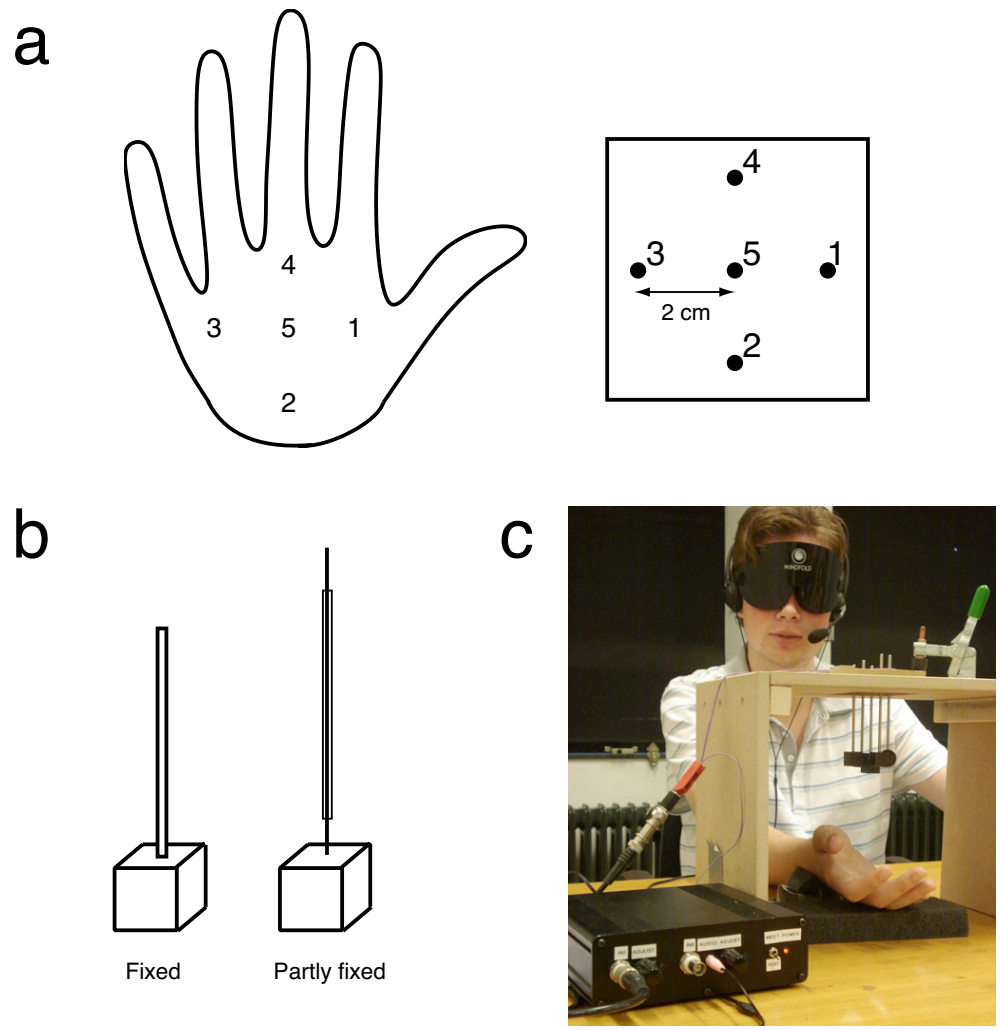

Figure 1: a) Shows a right hand with palm facing towards the front and the item positions are labelled according to the positions in the grid at which they could be fixed, shown on the right. b) Schematic drawing of the different methods of item fixation (in this case a cube). In the left-hand image the cube is rigidly fixed to a metal tube (fixed), while in the right-hand image the cube is fixed to a flexible wire which is pulled through a metal tube (partly fixed). The free wire end had a length of $0.5 \mathrm{~cm}$. c) A picture of a subject in the set-up.

with the centre item lower $(p<0.05)$ compared to the partly fixed conditions.

Response times for the different target positions are shown in Figure $2 \mathrm{~b}$. The target present trials were analysed further to investigate possible effects of target location. To this end, a $3 \times 2 \times 5$ (fixation method $\times$ target shape $\times$ target position) repeated measures ANOVA was performed on the response times from the target present trials. The ANOVA showed main effects for fixation method $\left(F_{2,14}=12.1, p<0.01\right)$, target shape $\left(F_{1,7}=38.9, p<\right.$ $0.001)$ and target position $\left(F_{4,28}=11.3, p<0.001\right)$. There were significant interaction effects between fixation method and target position $\left(F_{8,56}=4.2, p<0.001\right)$ and between target shape and target position $\left(F_{4,28}=5.6, p<0.01\right)$.

A post-hoc analysis was performed on the response times from the three methods of item fixation separately. Three separate $2 \times 5$ (target shape $\times$ target position) ANOVAs showed that for each of the methods of item fixation there was a main effect of target shape and target position. Only for the fixed-with-centre-item-lower condition was there a significant interaction between target shape and target condition. Bonferroni corrected post-hoc $t$-tests showed for the partially fixed condition that when the target was in the centre (position 5), the response time was significantly larger than when the target was near the thumb (position 1) $(p<0.05)$. For the fixed condition post-hoc $t$-tests showed that response times were significantly larger when the item was in the centre (position 5) than for each of the other positions $(p<0.01)$. For the fixed-with-centreitem-lower condition the interaction between target shape and target position was significant, therefore the analysis of differences between the positions was performed on the response times for the two target shapes separately. In this case no significant differences between the target positions were found.

\section{Discussion and Conclusion}

Our results show that constraining exploratory freedom by fixating the items does not necessarily yield longer search times. Allowing small displacements and rotation of the items (partially fixed) yielded longer response times than when the items were completely fixed to their location and orientation (fixed and fixed with centre item lowered). An explanation for this could be that it is easier to exert forces on the shapes when they are rigidly fixed. In this case search could be performed by grasping and squeezing the shapes. In a previous study where the shapes were suspended from wires and therefore not fixed to a position in the hand, target present response times found for five items were $720 \mathrm{~ms}$ for a cube among spheres and $830 \mathrm{~ms}$ for a sphere among cubes [14]. These response times are roughly in the same range as for the partially fixed condition $(690 \mathrm{~ms}$ and $1000 \mathrm{~ms})$, but larger than for the completely fixed conditions in the present study $(530 \mathrm{~ms}$ and $740 \mathrm{~ms}$ for fixed in the same plane and $500 \mathrm{~ms}$ and $700 \mathrm{~ms}$ for centre item lower). Response times from this previous study, however, should be only compared with caution since in that study the number of items was varied which may influence response times.

The general exploration method of grasping and squeezing the shapes simultaneously was observed for each of the experimental conditions. We chose to use spheres and cubes in this study, because it is known from a previous study that search for a cube among spheres and also the reverse can generally be performed through only grasping all of the shapes simultaneously [14]. In the same study it has been shown that in searches for other target shapes sometimes more detailed exploration is needed (e.g. when search- 
a

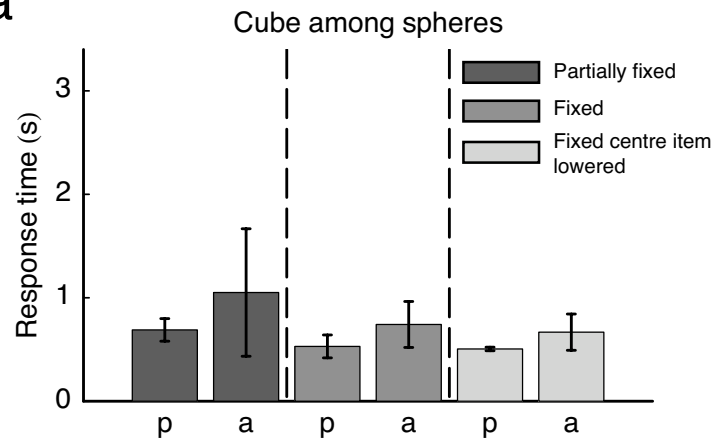

b

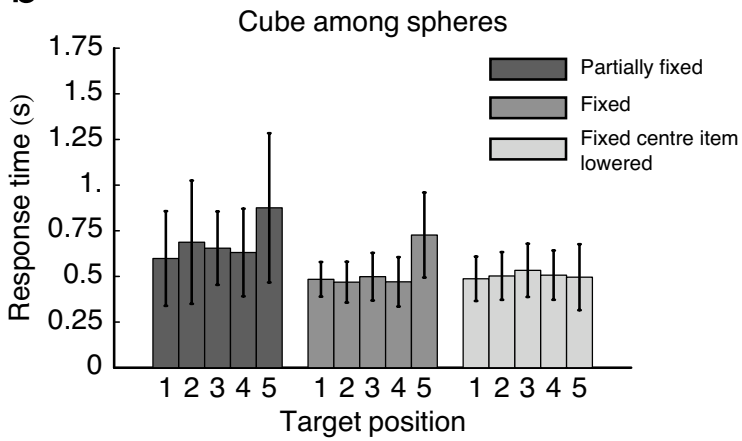

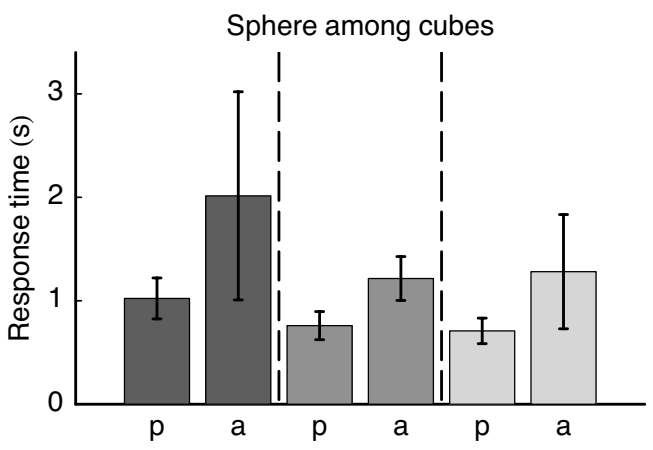

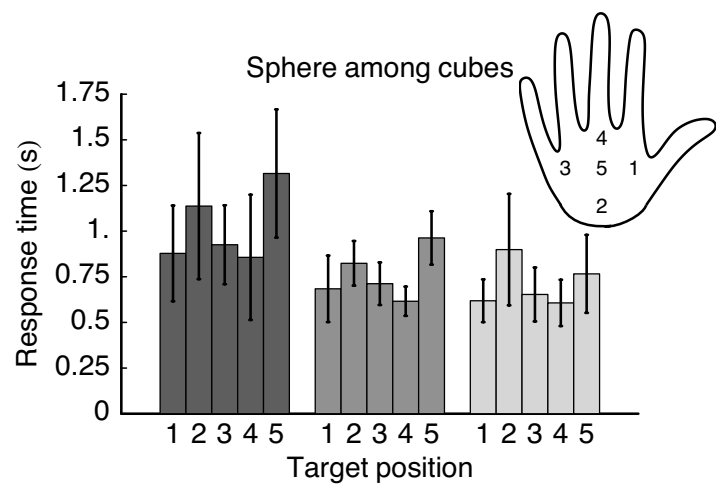

Figure 2: The left-hand panels show the results for search for a cube among spheres and the right-hand panels show results for search for a sphere among cubes. Error bars indicate the standard error of the mean. a) Response times averaged over subjects for target present, indicated with $\mathrm{p}$, and target absent trials, indicated with $\mathrm{a}$, in each of the item fixation methods. b) Response times for the different target positions.

ing for an ellipsoid among spheres). In such cases, it is possible that search is faster when items can be rotated or slightly translated. Item positions relative to the hand, however, are no longer constant during a trial for such serial and more time consuming exploratory strategies.

We have also shown that there are differences in search times for the different target locations. When all items were fixed in one plane, detection of a target in the centre was slower than for any of the other target positions. However, when the centre item was lowered as little as $0.5 \mathrm{~cm}$ there were no differences found for the response times between the different target positions. This shows that there are no large differences in response times for different positions in the hand, as long as skin contact can be easily made with each of the items. By lowering the centre item, the global shape of a grasping hand is followed and the centre item can be easily accessed.

The finding that response times are larger for search for a sphere among cubes than for a cube among spheres is in agreement with what we have reported in a previous study [14]. In that study cubes and spheres were suspended from wires and could be freely rearranged in the hand. Here it was also found that search for a cube among spheres is faster than the reverse condition. For that way of item presentation also sliding of the shapes along each other might have played a role. It is easier the rearrange a hand full of spheres than a hand full of cubes. This could also explain why search for a cube among spheres is faster than search for a sphere among cubes. As the items were (partially) fixed to a certain position in the present study, this factor could not play a role here. Consequently, the present study clearly supports the idea that detection of edges and vertices is faster than detection of curvature, or the absence of edges and vertices. Lederman and Klatzky [8] reported that search for the presence of an edge is faster than search for the absence of an edge. These findings support the idea that edges are highly salient features for haptic perception of shape.

An alternative explanation for search for a cube being faster than search for a sphere could involve heat conduction. Although the cube and the sphere had the same volume, they differed in surface area. The surface area of the cube is slightly larger than that of the sphere. The contact area with the skin was possibly larger for the cube resulting in more heat flow into the cube than into a sphere. However, contact area likely varied more with the position of the item in the hand than with its shape. Also skin temperature varies across different areas of the hand. Moreover, it has been shown that heat conductivity is not a very salient feature [8]. Therefore, it is much more likely that search for a cube was faster because it has edges and not because of heat conduction.

In conclusion, there are no large differences in search times for different target locations in the grasping hand as long as the skin contact can be easily made. Search for a cube among spheres is faster than search for a sphere among cubes also when the shapes are fixed to a certain spatial position or orientation. Finally, search for three-dimensional shape is faster when the shapes are rigidly fixed in space. This kind of fundamental knowledge about haptic perception can be useful in future design of especially hand worn haptic devices that allow for enclosing virtual objects with the hand.

\section{ACKNOWLEDGEMENTS}

This research was supported by a grant from the Netherlands Organisation for Scientific Research (NWO).

\section{References}

[1] Immersion Corporation: CyberGrasp. 
[2] S. Coren. The left-hander syndrome: The causes and consequences of left-handedness. New York: Vintage Books, 1993.

[3] J. C. Craig and K. B. Lyle. A comparison of tactile spatial sensitivity on the palm and fingerpad. Perception \& Psychophysics, 63:337-347, 2001.

[4] R. S. Johansson and A. B. Vallbo. Tactile sensibility in the human hand: Relative and absolute densities of four types of mechanoreceptive units in glabrous skin. Journal of Physiology, Vol. 286:283-300, 1979.

[5] S. J. Lederman, R. A. Browse, and R. L. Klatzky. Haptic processing of spatially distributed information. Perception \& Psychophysics, 44:222-232, 1988.

[6] S. J. Lederman and R. L. Klatzky. Hand movements: a window into haptic object recognition. Cognitive Psychology, 19:342-368, 1987.

[7] S. J. Lederman and R. L. Klatzky. Haptic classification of common objects: Knowledge-driven exploration. Cognitive Psychology, 22:421$459,1990$.

[8] S. J. Lederman and R. L. Klatzky. Relative availability of surface and object properties during early haptic processing. Journal of Experimental Psychology: Human Perception and Performance, 23:16801707, 1997.

[9] S. J. Lederman and R. L. Klatzky. Haptic identification of common objects: Effects of constraining the manual exploration process. Perception \& Psychophysics, 66:618-628, 2004.

[10] K. Minamizawa, S. Kamura, N. Kawakami, and S. Tachi. A palmworn haptic display for bimanual operations in virtual environments. In M. Ferre, editor, Haptics: Perception, devices and scenarios, volume 5024 of Lecture Notes on Computer Science, pages 458-463. Berlin/Heidelberg: Springer, 2008.

[11] K. E. Overvliet, K. M. Mayer, J. B. J. Smeets, and E. Brenner. Haptic search is more efficient when the stimulus can be interpreted as consisting of fewer items. Acta Psychologica, 127:51-56, 2008.

[12] M. A. Plaisier, W. M. Bergmann Tiest, and A. M. L. Kappers. Haptic pop-out in a hand sweep. Acta Psychologica, 128:368-377, 2008.

[13] M. A. Plaisier, W. M. Bergmann Tiest, and A. M. L. Kappers. Haptic search for spheres and cubes. In M. Ferre, editor, Haptics: Perception, devices and scenarios, volume 5024 of Lecture Notes on Computer Science, pages 275-282. Berlin/Heidelberg: Springer, 2008.

[14] M. A. Plaisier, W. M. Bergmann Tiest, and A. M. L. Kappers. Salient features in three-dimensional haptic shape perception. Perception \& Psychophysics, in press. 